\title{
Volcanic Ash and $\mathrm{SO}_{2}$ retrievals using synthetic MODIS TIR data: comparison between inversion procedures and sensitivity analysis
}

\author{
Stefano Corradini ${ }^{1 *}$, Sergio Pugnaghi ${ }^{2}$, Alessandro Piscini ${ }^{1}$, Lorenzo \\ Guerrieri $^{2}$, Luca Merucci ${ }^{1}$, Matteo Picchiani ${ }^{3}$, Marco ChinI ${ }^{4}$ \\ 1 Istituto Nazionale di Geofisica e Vulcanologia, Centro Nazio- \\ nale Terremoti, Roma, Italy, 2 Università degli Studi di Modena e \\ Reggio Emilia, Dipartimento di Scienze Chimiche e Geologiche, \\ Modena, Italy, 3 Tor Vergata University, Rome, Italy, 4 Luxem- \\ bourg Institute of Science and Technology, Environmental Re- \\ search and Innovation Department, Belvaux, Luxembourg \\ *stefano.corradini@ingv.it
}

\begin{abstract}
In this work the volcanic ash and $\mathrm{SO}_{2}$ retrievals obtained by applying three different procedures (LUT - Look Up Table, NN - Neural Network and VPR - Volcanic Plume Removal) on MODIS Thermal InfraRed (TIR) synthetic measurements have been compared. The synthetic measurements are generated using MODTRAN Radiative Transfer Model (RTM) for defined volcanic cloud configurations. The results, presented as the percentage difference between the retrieved ash and $\mathrm{SO}_{2}$ total masses and the true values used for the synthetic data generation, indicate maximum differences of $+/-15 \%$ and $+/-10 \%$ for all the procedures and for ash and $\mathrm{SO}_{2}$ retrievals respectively. A sensitivity analysis has been also realized to investigate the influence of volcanic cloud altitude and water vapour profile on $\mathrm{SO}_{2}$ retrievals at 7.3 and $8.6 \mu \mathrm{m}$. Results confirm the high sensitivity of the $7.3 \mu \mathrm{m}$ retrieval to the volcanic cloud altitude and show that the $\mathrm{SO}_{2}$ total masses estimated at 7.3 and $8.6 \mu \mathrm{m}$ separately can be used to improve the information on the plume height. Finally, the water vapour profile is used to compute the minimum altitude over which the $7.3 \mu \mathrm{m}$ retrieval is effective.
\end{abstract}

\section{INTRODUCTION}

$\mathrm{T}$ he 2010 Eyjafjallajokull eruption made evident the need to obtain more accurate volcanic ash and $\mathrm{SO}_{2}$ quantitative estimations in a near real time basis [Zenher, 2010]. To answer this pressing requirements the scientific community is forced to develop novel, fast and reliable retrieval approaches. In this paper the volcanic ash and $\mathrm{SO}_{2}$ retrievals are realized by using the well established LUT (Look Up Table) procedure [Corradini et al., 2009], and the novel VPR (Volcanic Plume Removal) [Pugnaghi et al., 2013] and NN (Neural Network) approaches [Picchiani et al., 2011; Piscini et al., 2014]. The results are compared 
ANNALS OF GEOPHYSICS, Fast Track 2, 2014

and discussed and will give us information on the reliability of the inversion schemes presented. As test cases different MODIS (Moderate Resolution Imaging Spectroradiometer) TIR (Thermal InfraRed) synthetic measurements have been generated using MODTRAN Radiative Transfer Model (RTM) considering defined volcanic cloud configurations. A sensitivity analysis has also been realized to investigate the influence of volcanic cloud altitude uncertainty and the effect of the atmospheric water vapour $(\mathrm{Wv})$ profile on $\mathrm{SO}_{2}$ retrieval.

The paper is organized as follows: in Sections II and III the retrieval procedures and the synthetic MODIS data generation are described. Section IV shows the results of the ash and $\mathrm{SO}_{2}$ retrievals comparison and in Section $\mathrm{V}$ the sensitivity analysis is realized. The conclusions are drafted in Section VI.

\section{Retrievals Procedure Description}

The LUT, VPR and NN procedures have been designed for the simultaneous volcanic ash and $\mathrm{SO}_{2}$ retrievals using multispectral satellite data in the TIR spectral range. In this work the different approaches have been applied to MODIS synthetic measurements. MODIS, aboard the NASA Terra and Aqua polar satellites with a repetition cycle of 1 to 2 days, acquire data in 36 bands from VIS to TIR with a spatial resolution of $1 \mathrm{~km}^{2}$ [http://modis.gsfc.nasa.gov/]. The TIR channels 28-29 (centered at 7.3 and $8.6 \mu \mathrm{m}$ ) and 31-32 (centered at 11 and $12 \mu \mathrm{m}$ ) are used for the volcanic $\mathrm{SO}_{2}$ and ash retrievals respectively. In the following the retrieval approaches considered are described.

- LUT approach: the ash detection and retrievals (aerosol optical depth $-\tau$, effective radius $r_{e}$ and total mass $-m_{t}$ ) are based on the exploitation of the different spectral ash signature around 11 and $12 \mu \mathrm{m}$. The $\mathrm{SO}_{2}$ retrievals are realized considering the two wide spectral signature around 7.3 and $8.6 \mu \mathrm{m}$ and using a $\chi^{2}$ procedure. The ash effect on $\mathrm{SO}_{2}$ estimations has been taken into account considering the correction procedure developed by Corradini et al., [2009]. All the retrievals needed RTM simulations computed for specific atmospheric profiles, volcanic cloud geometry, surface parameters and ash type. The main advantage of the LUT approach is the precise characterization of the local atmosphere while the main drawback is the non-real time processing.

- VPR approach: by linearly interpolating the radiances surrounding a detected volcanic cloud, the VPR procedure computes the radiances that would have been measured by the sensor in the absence of the volcanic cloud itself, and reconstructs a new image without it. The new image and the original data allow the computation of the plume transmittance in the MODIS TIR bands $29\left(t_{29}\right), 31\left(t_{31}\right)$, and $32\left(t_{32}\right)$ by applying a simplified model consisting of a plume at a constant altitude, temperature and thickness. The transmittances are then refined with a polynomial relationship obtained by means of MODTRAN simulations adapted for the geographical region, ash type, and atmospheric profiles. $\tau, r_{e}$ and $m_{t}$ are computed from $t_{31}$ and $t_{32}$ while $\mathrm{SO}_{2}$ is retrieved from $t_{29}$ and $t_{31}$. The main advantages of VPR is that it works in real time and that, once the polynomial coefficients are computed (for a specific volcano, ash type and sensor), the volcanic cloud altitude and temperature are the only input parameters required to run the procedure. The main drawback is that the surface under the volcanic cloud should be homogeneous enough.

- NN approach: is based on the coefficients computation of the relationship existing between the Top Of Atmosphere (TOA) spectral radiance and the volcanic ash and $\mathrm{SO}_{2}$ parameters. The NN coefficients estimation is realized in the learning phase by examples 
composed of training patterns, i.e. MODIS spectral radiances samples, and the corresponding ash $\left(\tau, r_{e}, m_{t}\right)$ and $\mathrm{SO}_{2}$ values. The topologies for the ash and $\mathrm{SO}_{2}$ parameters estimations are established using the MODIS TIR channels 28-31-32 and 29-31-32 as inputs and ten neurons for the single hidden layer of each NN. The main advantage of the NN procedure is the real time processing while the main drawback is that it requires a good training samples statistical distribution.

\section{MODIS SYNTHETIC MEASUREMENTS GENERATION}

The use of synthetic data instead of real measurements is due to the need to test the inversion schemes on known volcanic cloud configurations. The ash and $\mathrm{SO}_{2}$ retrieved from the different approaches can be compared directly with the 'true' values used for the MODIS TIR synthetic data generation. These images are produced by using the MODTRAN RTM, for the MODIS TIR channels 28, 29, 31 and 32, and considering specific surface characteristics, atmospheric profiles, plume geometry, ash type and different quantities of ash and $\mathrm{SO}_{2}$. The volcanic cloud configurations are representative of Mt. Etna (Sicily-Italy) emissions. As atmospheric profiles (Pressure, Temperature, Water vapour - PTWv) the Trapani WMO data collected the 23 October at 12:00 UTC, the 24 October 2011 at 00:00 UTC and the 15 November 2011 at 12:00 UTC have been considered. These profiles have been selected because representative of different $W v$ content (31, 23 and $15 \mathrm{~mm}$ respectively). The volcanic cloud top altitudes has been set to 5,6 and $7 \mathrm{~km}$ with 1 $\mathrm{km}$ of thickness and as ash type, the Volz [1973] ash refractive index has been chosen.
The volcanic cloud shape is considered ellipsoidal, the ash $\tau$ and $\mathrm{SO}_{2}$ distributions are Gaussian with peaks at 1.25 and $10 \mathrm{~g} / \mathrm{m}^{2}$ respectively in different positions with different standard deviations. The effective radii are randomly distributed with values equally spaced in a logarithmic scale from 0.8 to 10 $\mu \mathrm{m}$. Figure 1 shows the ash $\tau, r_{e}$ and $\mathrm{SO}_{2}$ distribution used for the generation of the 9 test cases (3 atmospheric profiles and 3 volcanic cloud altitudes). Finally, the View Zenith Angle (VZA) of the synthetic image vary between $22^{\circ}$ and $34^{\circ}$ (typical for MODIS images collected on Etna volcano).

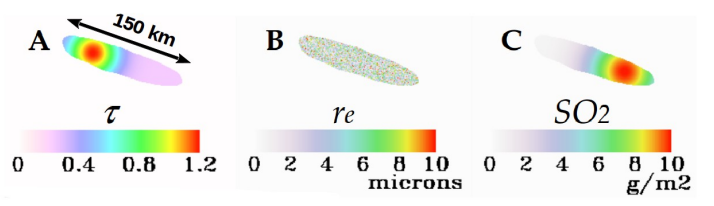

Figure 1: Volcanic cloud configuration. $\tau$ (plate A), $r_{e}$ (plate $\mathrm{B}$ ) and $\mathrm{SO}_{2}$ (plate C).

For each of the 9 test cases (named in the following 'standard' test cases), a perturbation has been also applied to take into account the MODIS TOA radiance variability of the real images due to change in surface temperature, emissivity and path radiance. For each pixel, the perturbed radiance at the sensor is a random number of normal distribution with mean equal to the radiance without perturbation and standard deviation corresponding to the $0.5 \%$ of the same value. These cases will be named in the following 'perturbed' test cases.

The LUT procedure runs with the same MODTRAN simulations used to generate the synthetic measurements, the VPR is applied by knowing the right volcanic cloud altitude and temperature and the NN is trained by considering the $50 \%$ of the volcanic cloud of each im- 
age and tested on the remaining 50\%. Therefore, because no uncertainties can be attributed to the input parameters, the discrepancy between the retrieved ash and $\mathrm{SO}_{2}$ masses and the truth, for the standard images, represents the intrinsic error of the linearization process for the different retrieval schemes. The tests realized by using the 'perturbed' images will give us the sensitivity of the inversion schemes on the TOA radiance variability.

\section{RESULTS}

The results are presented as the percentage difference between the total ash and $\mathrm{SO}_{2}$ masses retrieved $\left(\mathrm{M}_{\text {retr }}\right)$ and the 'true' values $\left(\mathrm{M}_{\text {true }}\right)$ used to generate the MODIS synthetic images $\left(\mathrm{D} \%=100 *\left[\mathrm{M}_{\text {retr }}-\mathrm{M}_{\text {true }}\right] / \mathrm{M}_{\text {true }}\right)$.

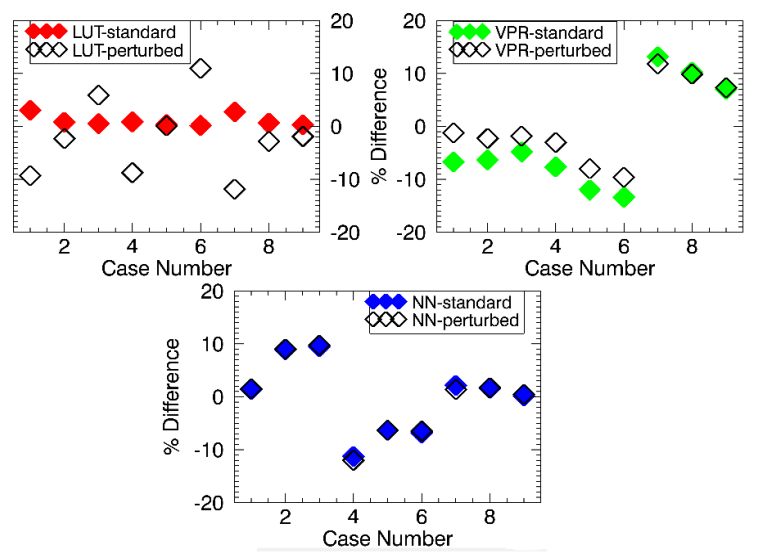

Figure 2: Ash mass percentage differences. LUT (top left), VPR (top right) and NN (bottom) retrievals.

In Figure 2 are shown the results for the ash retrievals obtained from the different procedures and for all the 18 test cases. The LUT percentage difference (top left plate) is limited to $5 \%$ for the standard cases and increases to about $10 \%$ for the perturbed cases. The VPR differences (top right plate of Figure 2) are all within $+/-15 \%$ and are not affected by the TOA radiance perturbation. As VPR also the NN results are not affected by the perturbations and the \% differences reach about the $+/-10 \%$. Figure 3 shows the results for the $\mathrm{SO}_{2}$ retrievals obtained from all the procedures for all the test cases. As the top plates show the LUT retrievals have been realized by considering both the 7.3 and $8.6 \mu \mathrm{m}$ MODIS channels separately.

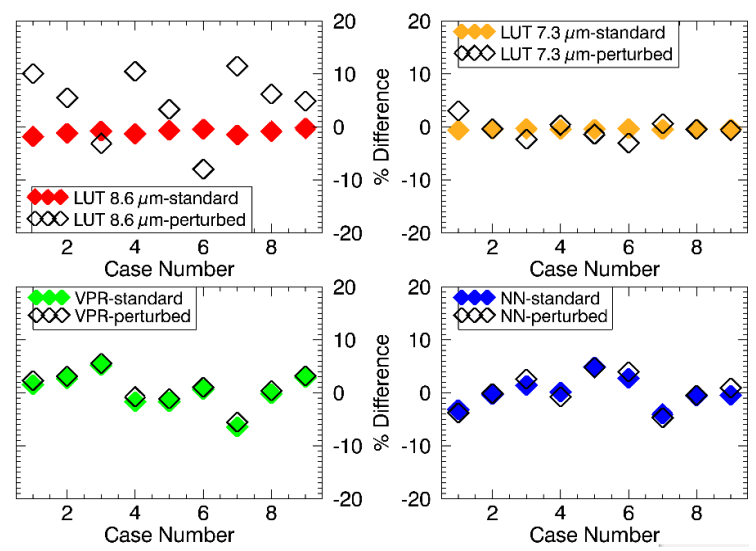

Figure 3: $\mathrm{SO}_{2}$ mass percentage differences. Top plates from left to right: LUT $8.6 \mu \mathrm{m}$ and LUT $7.3 \mu \mathrm{m}$ retrievals. Bottom plates from left to right: VPR and NN retrievals.

For both the LUT retrievals the percentage difference is limited to $+/-5 \%$ for all the standard cases. It increases until $+/-10 \%$ for the $8.6 \mu \mathrm{m}$ considering the perturbed test cases. As for ash the VPR and $\mathrm{NN} \mathrm{SO}_{2}$ retrievals are not affected by the TOA radiance perturbation and the mass differences are all within the $+/-10 \%$. As Figures 2 and 3 show, and as expected, the lower differences are obtained with the LUT inversion for the 'standard' cases. The perturbation increases meaningfully the discrepancies. On the contrary the VPR and NN inversions give approximately the same results for both standard and perturbed test cases. These latter are simplified algorithms and consequently they give less accurate results compared to LUT in the standard cases. But at the same time, VPR and NN are less sensitive to small radiance variations introduced in the 'perturbed' cases. 


\section{SENSITIVITY ANALYSIS FOR $\mathrm{SO}_{2}$ RETRIEVALS}

The results shown in figure 3 for the $\mathrm{SO}_{2} \mathrm{LUT}$ retrievals at $7.3 \mu \mathrm{m}$ can be misleading. Due to the strong water vapour absorption around 7.3 $\mu \mathrm{m}$, the $\mathrm{SO}_{2}$ estimation on this channels is strongly dependent on the $W v$ profile then on volcanic cloud altitude [Corradini et al., 2009; 2010]. For all the previous test cases these two parameters were completely known (see Section III). To quantify the $\mathrm{SO}_{2}$ retrieval error due to the uncertainty on volcanic cloud altitude the LUT procedure has been applied considering a specific synthetic image (generated using the 24 October 2011 PTH profiles and a plume altitude of $6 \mathrm{~km}$ ), and processed using different volcanic cloud altitudes. Figure 4 shows the percentage differences between true and retrieved $\mathrm{SO}_{2}$ values at 7.3 and $8.6 \mu \mathrm{m}$ by considering the volcanic cloud at 4, 5, 5.5, 6, 6.5, 7 and $8 \mathrm{~km}$. As can be seen, an uncertainty of $500 \mathrm{~m}$ on the plume altitude leads to an error on $\mathrm{SO}_{2}$ retrievals of about the $50 \%$ and $10 \%$ for 7.3 and $8.6 \mu \mathrm{m}$ retrievals respectively (a similar trend can be also found in Campion et al., 2010, see Figure 4). The analysis emphasize also that the two retrievals give the same results for the correct volcanic cloud altitude. When the volcanic cloud altitude considered for the retrievals is greater than the true value the $7.3 \mu \mathrm{m}$ retrievals is always lower than the $8.6 \mu \mathrm{m}$ retrieval and vice versa. The knowledge of the $W v$ profile can also be used to investigate the minimum volcanic cloud altitude over which the $7.3 \mathrm{SO}_{2}$ retrieval is effective.

To do it the Noise Equivalent Spectral Radiance - NESR (i.e. the minimum radiance variation detectable by the sensor) for the MODIS channel 28 , is compared with the TOA radiance variations induced by different values of
$\mathrm{SO}_{2}$ abundance for volcanic cloud at different altitudes.

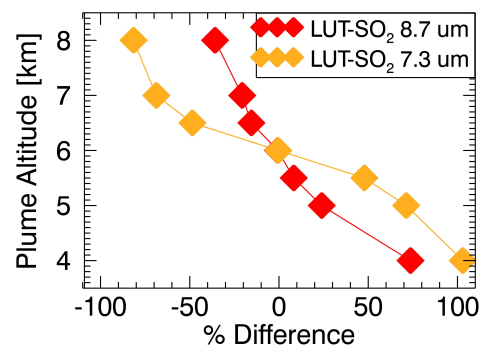

Figure 4: $\mathrm{SO}_{2}$ retrievals at 7.3 and $8.7 \mu \mathrm{m}$ : Percentage differences between true and retrieved values for volcanic clouds at different altitudes.

These latter values are computed from the difference between the MODIS channel 28 TOA radiance in a free atmosphere $\left(L_{0}\right)$ and the TOA radiance considering a volcanic cloud at different altitudes with different values of columnar abundance $L_{s}\left(C_{s}\right)$. Instead of the vertical column density $(W v)$ more significant is the slant column density $\left(W v^{*}\right)$ that takes into account the VZA i.e. the real path crossed by the atmospheric radiation $\left(W v^{*}=W v / \cos (V Z A)\right)$. Figure 5 shows the 3D plots of $L_{0}-L_{s}\left(C_{s}\right)$ for the atmospheric profiles of 23 (top plates) and 24 (bottom plates) October 2011, volcanic cloud at 4 $\mathrm{km}$ and considering mean VZA equal to $28^{\circ}$ (left plates) and $50^{\circ}$ (right plates). The horizontal flat surface is the NESR threshold $\left(0.0173 \mathrm{~W} / \mathrm{m}^{2} / \mathrm{sr} / \mu \mathrm{m}\right)$. As figure shows for higher $W v^{*}$ and lower volcanic cloud altitudes (upper right plate), the most of the $\mathrm{SO}_{2}$ is undetectable. For a volcanic cloud at the same altitude and lower $W v^{*}$ value (bottom plates) the $\mathrm{SO}_{2}$ is almost all detectable.

\section{Summary AND CONCLUSION}

In this work the volcanic ash and $\mathrm{SO}_{2}$ estimations are realized by using different retrieval 
approaches applied to MODIS TIR synthetic data. Because no uncertainties can be attributed to the input parameters, for the standard images the discrepancy between the retrieved and true values represent the intrinsic error of the linearization process of the different retrieval schemes.

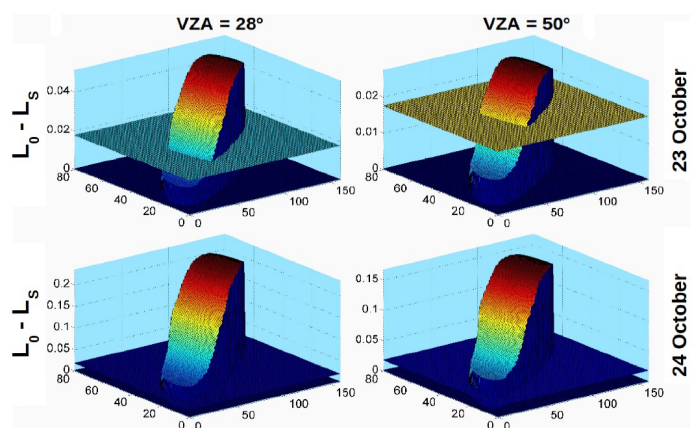

Figure 5: $3 D$ plots of $L_{0}-L_{s}\left(c_{s}\right)$ at $7.3 \mu \mathrm{m}$ for different atmospheric profiles and VZA. The horizontal flat surface is the $7.3 \mu \mathrm{m}$ NESR. In ordinate and abscissa the pixel number.

Results indicate that the maximum discrepancy between retrievals and truth are all within $+/-15 \%$ and $+/-10 \%$ for all the procedures and for ash and $\mathrm{SO}_{2}$ retrievals respectively. The data perturbation affects significantly only the LUT retrievals. The sensitivity analysis shows that the the 7.3 and $8.6 \mu \mathrm{m} \mathrm{SO}_{2}$ retrievals realized separately can improve the information on volcanic cloud altitude. Finally, it is shown how to use the water vapour profile to compute the minimum volcanic cloud altitude over which the $7.3 \mu \mathrm{m}$ retrieval is effective.

\section{ACKNOWLEDGEMETS}

The work of S. Corradini was founded by the European Union's Seventh Framework Programme (FP7/2007-2013) through the project APhoRISM under grant agreement n. 606738.
[Campion et al., 2010] Campion, R., Salerno, G. et al. (2010). Measuring volcanic degassing of $\mathrm{SO} 2$ in the lower troposphere with ASTER band ratios, JVGR, 194, 42-54.

[Corradini et al., 2009] Corradini, S., Merucci, L., and Prata, A. J. (2009). Retrieval of $\mathrm{SO}_{2}$ from Thermal Infrared Satellite Measurements: Correction Procedures for the Effects of Volcanic Ash, AMT, 2, 177-191.

[Picchiani et al., 2011] Picchiani, M., Chini, M., et al. (2011). Volcanic ash detection and retrievals from MODIS data by means of Neural Networks, AMT, 4, 2619-2631, 2011.

[Piscini et al., 2014] Piscini, A., Picchiani, M., Chini, M., et al. (2014) A Neural Network approach for the simultaneous retrieval of volcanic ash parameters and $\mathrm{SO}_{2}$ using MODIS data, AMTD-2014-57.

[Pugnaghi et al., 2013] Pugnaghi, S., Guerrieri, L., Corradini, S., et al.. (2013). A new simplified procedure for the simultaneous $\mathrm{SO}_{2}$ and ash retrieval in a tropospheric volcanic cloud, AMT, 6, 1315-1327

[Volz, 1973] Volz, F. E. (1973). Infrared optical constants of ammonium sulfate, Sahara dust, volcanic pumice and fly ash, AO, 12, 564-568. [Zenher, 2010] Zehner, C. (2010). Monitoring Volcanic Ash from Space. Proceedings of the ESA-EUMETSAT workshop on the 14 April to 23 May 2010 eruption at the Eyjafjoll volcano, South Iceland. Frascati, Italy, 26-27 May 2010. ESA-Publication STM-280.

\section{REFERENCES}

